\title{
Biologic Prescribing Patterns Among Mount Sinai Psoriasis Patients: Results of a Retrospective Chart Review
}

\author{
Alexa Choy, ${ }^{1}$ Jonathan Vebman, ${ }^{1}$ Christopher J. Yao, $\mathrm{MPH}^{1,2}$ \\ ${ }^{1}$ Department of Dermatology, Icahn School of Medicine at Mount Sinai, New York, NY \\ ${ }^{2}$ University of Rochester School of Medicine and Dentistry, Rochester, NY
}

\section{ABSTRACT}

Introduction: Psoriasis is a painful and chronic inflammatory skin condition that not only impacts the quality of life of patients but is also a socioeconomic burden due to the cost of treatment, particularly with biologic treatments.

Objective: The purpose of the study is to understand biologic prescribing patterns among Mount Sinai psoriasis patients and assess its relationship to insurance policy, which may limit treatment access.

Methods: This study reviewed randomized, de-identified charts of psoriasis patients in a nine-physician academic practice at Mount Sinai (New York, United States) with the following insurers: Aetna, Blue Cross Blue Shield, Empire Blue Cross Blue Shield, Medicare A\&B, and United Healthcare, treated with the following biologics: secukinumab, etanercept, adalimumab, infliximab, brodalumab, ustekinumab, ixekizumab and guselkumab. A chisquare test was performed to compare prescribed biologics by insurance company.

Results: Ustekinumab was the most prescribed biologic treatment across all the insurance plans. There were also disproportionate prescriptions of certain biologics for patients under particular insurance plans. Etanercept, brodalumab, and infliximab were the least prescribed biologics.

Conclusion: Results highlight certain patterns in the prescribed biologics of Mount Sinai patients. Prescribed biologics tend to vary by different insurers. However, ustekinumab was the most frequently prescribed biologic among all insurers, though it is not the most efficacious biologic based on PASI responses. Future research should be conducted to assess how differences in insurance policies (e.g. cost to patient) affect biologic prescribing.

\section{INTRODUCTION}

Psoriasis is a painful and chronic inflammatory skin condition that can be disabling to both the physical and psychological health of affected patients. ${ }^{1}$ In 2013, it was reported psoriasis was prevalent in 7.4 million adults in the United 
States. ${ }^{2}$ Psoriasis is also associated with an increased risk of comorbidities, such as psoriatic arthritis, Crohn's disease, malignancy, psychological/mental illness, obesity and cardiovascular diseases. ${ }^{3}$ There is also a significant socioeconomic burden in the cost of psoriasis and its treatments. ${ }^{1}$ Among U.S. adults, $32.1 \%$ of those with restricted prescription medication, experienced a prominent decline in their health status. ${ }^{4}$ Psoriasis currently has no cure and, therefore, requires extensive and long-term treatment. ${ }^{1}$

Though a common and effective treatment for psoriasis, biologic therapies have higher costs, compared to other psoriasis treatments, such as phototherapy and traditional systematic treatments. ${ }^{10}$ There are also differences in efficacy among the biologics. A recent network meta-analysis showed brodalumab was significantly more efficacious than secukinumab, ustekinumab and etanercept based on PASI responses after 52 weeks. ${ }^{11}$ However, little information concerning insurance policy and access to biologics is known. One recent survey in ulcerative colitis found that $219(43.8 \%)$ of surveyed gastroenterologists experienced limitations to prescribing biologics. Two of the most commonly cited prescribing barriers included patient insurance restrictions (79.0\%) and out-of-pocket costs $(71.7 \%) .{ }^{12}$ Thus, the purpose of this study is to understand biologic prescribing patterns among Mount Sinai psoriasis patients and assess its relationship to insurance policy, which may limit treatment access.

\section{METHODS}

\section{Chart Review}

This retrospective study was conducted on randomized de-identified charts of psoriasis patients from the medical electronic billing database, Epic, at the Mount Sinai Dermatology Department faculty practice, from nine different dermatologists. The charts were dated from December 5, 2016 through June 25, 2018. The conducted review collected charts of psoriasis patients with the highest frequency insurance plans at Mount Sinai's Dermatology Department: Aetna, Blue Cross Blue Shield (BC/BS), Empire Blue Cross Blue Shield (Empire BC/BS), Medicare A\&B and United Healthcare, treated with the following biologic treatments: ustekinumab, guselkumab, adalimumab, secukinumab, ixekizumab, etanercept, brodalumab and infliximab. Data were collected on patient's sex, age, zip code, insurance company and prescribed biologic.

\section{Statistical Analysis}

Statistical analyses were conducted with Stata Version 15.1 (StataCorp LLC, College Station, TX, USA). First, we generated demographic statistics (age, sex, residence) for patients with each insurance company. A contingency table chi-square test was performed for patients with the insurance plans Aetna, BC/BS, Empire BC/BS, Medicare A\&B, and United Healthcare and prescribed either adalimumab, ustekinumab or guselkumab. Secukinumab, etanercept, infliximab, brodalumab and ixekizumab were omitted from this contingency table chisquare test because fewer than 5 patients had these combinations: Empire BC/BS with secukinumab, BC/BS with etanercept, Empire BCBS with etanercept, Aetna with infliximab, Empire BC/BS with infliximab, Medicare A\&B with infliximab, Aetna with brodalumab, BC/BS with brodalumab, Empire BC/BS with brodalumab, United Healthcare with brodalumab, BC/BS with ixekizumab and Empire BC/BS with ixekizumab. 


\section{RESULTS}

\section{Patient Sample}

The study cohort included 210 unique patients with 579 prescriptions for a biologic treatment from their dermatologists (Table 1). Ninety-three patients (44.3\%) were female and 117 (55.7\%) were male. Seventy-four patients $(35.2 \%)$ resided in Manhattan, $28(13.3 \%)$ in New Jersey and $27(12.9 \%)$ in Brooklyn, while the remaining 81 patients $(38.6 \%)$ resided in the greater New York area or other regions of the United States. The mean age (standard deviation) was 53.0 (19.3) with the majority $(21.4 \%)$ within the age range 61-70.

\section{Biologics prescribed for patients on different insurance plans}

Overall, ustekinumab was the most commonly prescribed biologic $(n=276$ [47.7\%]) (Table 2). Ustekinumab was also the most frequently prescribed biologic for each individual insurance plan: Aetna (52.0\%), BC/BS (61.5\%), Empire BC/BS (34.7\%), Medicare A\&B (43.9\%) and United Healthcare (44.1\%) (Table 2).

There was also a disproportionate amount of prescriptions for particular biologic treatments for some insurers. Of the Empire $\mathrm{BC} / \mathrm{BS}$ patients, there were $24.5 \%$ adalimumab and $26.5 \%$ guselkumab prescriptions; only $8.2 \%$ secukinumab, $4.1 \%$ ixekizumab and 2.0\% infliximab prescriptions, and no prescriptions for brodalumab. For United Healthcare patients, $22.1 \%$ of patients were prescribed secukinumab, compared to fewer than $10 \%$ of prescriptions for other biologics. Infliximab, brodalumab and etanercept were the least prescribed biologics at the respective frequencies: 12 (2.1\%), $17(2.9 \%)$ and $26(4.5 \%)$ (Table 2). For patients with Aetna, BC/BS, Empire BC/BS, Medicare $A \& B$ and United Healthcare and prescribed either ustekinumab, guselkumab or adalimumab, there was a significant difference between the number of patients with the insurance plans and their biologic prescription $(\mathrm{p}<0.001)$.

Table 1. Descriptive statistics of analyzed patients $(n=210)$.

\begin{tabular}{|ll|}
\hline Variable & Frequency (\%) \\
\hline Sex \\
\hline \multicolumn{1}{|l|}{ Female } \\
\hline Male & $93(44.3)$ \\
\hline Age (mean \pm SD) & $117(55.7)$ \\
\hline Age ranges & $54.0 \pm 19.3$ \\
\hline $\mathbf{1 1 - 2 0}$ & \\
\hline $\mathbf{2 1 - 3 0}$ & \\
\hline $\mathbf{3 1 - 4 0}$ & $6(2.9)$ \\
\hline $\mathbf{4 1 - 5 0}$ & $19(9.1)$ \\
\hline $\mathbf{5 1 - 6 0}$ & $43(20.5)$ \\
\hline $\mathbf{6 1 - 7 0}$ & $22(10.5)$ \\
\hline $\mathbf{7 1 - 8 0}$ & $28(13.3)$ \\
\hline $\mathbf{8 1 - 9 0}$ & $45(21.4)$ \\
\hline $\mathbf{9 0 - 1 0 0}$ & $30(14.3)$ \\
\hline Region by zip code & $16(7.6)$ \\
\hline Manhattan & $1(0.5)$ \\
\hline New Jersey & \\
\hline Brooklyn & \\
\hline Other & $74(35.2)$ \\
\hline & $28(13.3)$ \\
\hline & $27(12.9)$ \\
\hline
\end{tabular}


Table 2. Frequency (\%) of biologics prescribed for patients on different insurance plans* Insurance companies studies were: Aetna, Blue Cross Blue Shield (BC/BS), Empire Blue Cross Blue Shield (Empire BC/BS), Medicare A\&B and United Healthcare with the biologics: ustekinumab, guselkumab, adalimumab, secukinumab, ixekizumab, etanercept, brodalumab and infliximab. The contingency table chi-square test for performed for patients with the insurance plans Aetna, BC/BS, Empire BC/BS, Medicare A\&B and United Healthcare and prescribed either ustekinumab, guselkumab or adalimumab was statistically significant, indicating there is a difference between number of patients with the aforementioned insurance plans and their biologic prescription $(p<0.001)$.

\begin{tabular}{|lllllll|}
\hline \multicolumn{7}{c|}{ Insurance Company (\%) } \\
\hline Biologic & Aetna & BC/BS & Empire BC/BS & Medicare & United & Total \\
\hline Ustekinumab & $64(52.0)$ & $56(61.5)$ & $17(34.7)$ & $83(43.9)$ & $56(44.1)$ & $276(47.7)$ \\
\hline Guselkumab & $19(15.5)$ & $7(7.7)$ & $13(26.5)$ & $23(12.2)$ & $11(8.7)$ & $73(12.6)$ \\
\hline Adalimumab & $9(7.3)$ & $8(8.8)$ & $12(24.5)$ & $16(8.5)$ & $9(7.1)$ & $54(9.3)$ \\
\hline Secukinumab & $7(5.7)$ & $9(9.9)$ & $4(8.2)$ & $22(11.6)$ & $28(22.1)$ & $70(12.1)$ \\
\hline Ixekizumab & $13(10.6)$ & $4(4.4)$ & $2(4.1)$ & $24(12.7)$ & $8(6.3)$ & $51(8.8)$ \\
\hline Etanercept & $8(6.5)$ & $0(0.0)$ & $0(0.0)$ & $10(5.3)$ & $8(6.3)$ & $26(4.5)$ \\
\hline Brodalumab & $3(2.4)$ & $1(1.1)$ & $0(0.0)$ & $10(5.3)$ & $3(2.4)$ & $17(2.9)$ \\
\hline Infliximab & $0(0.0)$ & $6(6.6)$ & $1(2.0)$ & $1(0.5)$ & $4(3.2)$ & $12(2.1)$ \\
\hline Total (\%) & $123(100.0)$ & $91(100.0)$ & $49(100.0)$ & $189(100.0)$ & $127(100.0)$ & $579(100.0)$ \\
\hline
\end{tabular}

${ }^{*}$ Specific insurance information for each plan was not available to reference specific policies for biologic access.

\section{DISCUSSION}

\section{High number of prescriptions}

Though this study originally intended to identify prescribing patterns among different insurers, the most significant outcome was identifying ustekinumab as the most frequently prescribed biologic among all insurers and within the entire cohort. Interestingly, ustekinumab is not as effective as some other biologics. For instance, Yao and Lebwohl (2019) analyzed the time of onset of antipsoriatic drugs, including popular, available biologics for psoriasis. ${ }^{5}$ That study analyzed two outcomes: time for $25 \%$ of patients to achieve a $75 \%$ improvement from baseline PASI (PASI 75) and time for patients to achieve a mean $50 \%$ improvement from baseline PASI (PASI 50). Ustekinumab performed slower than brodalumab, ixekizumab, secukinumab, infliximab, and adalimumab in both study outcomes. Additionally, results from a longterm (52 week) efficacy meta-analysis of biologic PASI responses also determined brodalumab and secukinumab both responded with higher proportions of PASI 75, PASI 90 and PASI 100 than ustekinumab. ${ }^{11}$

Though ustekinumab is a less efficacious biologic treatment compared to some of the other common antipsoriatic biologics, it is the most frequently prescribed within this study cohort. At Mount Sinai, because ustekinumab is only prescribed every 3 months, it is administered during the patients' office visit and therefore qualifies as a medical benefit. Because most patients have co-insurance, with supplementary insurance to pay their copayments, their inMarch 2020 Volume 4 Issue 2 
office treatments are covered as part of the office visit and therefore the patient has no out-of-pocket expenses. However, the other biologics are considered a pharmacy benefit because they are self-administered; therefore, the patient must pay a copayment out-of-pocket every time their prescription is renewed. Thus, because ustekinumab may end up being less costly for the patient due to these insurance policies, doctors may choose to prescribe it more often.

\section{Low number of prescriptions}

The low number of prescriptions for infliximab, brodalumab and etanercept was also examined. Although specific reasons for biologic selection was not identified in the charts, potential explanations are plausible. Infliximab is given by infusion and therefore may be unfavorable to dermatologists. It is also plausible brodalumab is not often prescribed by dermatologists because of the package insert stating brodalumab is associated with suicidal ideations. ${ }^{6}$ Etanercept was likely limited in prescriptions because of its lower efficacy relative to other agents. Low and high doses of etanercept are the slowest acting antipsoriatic biologics compared to secukinumab, etanercept, adalimumab, infliximab, brodalumab, ustekinumab, and ixekizumab, making it the least effective of the all the biologics in this study. ${ }^{5}$

\section{Limitations}

There are limitations to this study. Data only included Mount Sinai patients and were therefore not representative of other hospitals or regions. Mount Sinai Dermatology faculty practice also does not include Medicaid patients, who are seen in the resident clinic; therefore, the results of the study do not factor in the Medicaid population. It is also not definitive whether a dermatologist prescribed a certain drug due to insurance coverage restrictions or for other reasons. For example, pre-existing, chronic co-morbidities could be a determining factor for why a patient would be prescribed a particular biologic (and not necessarily the most efficacious biologic). ${ }^{9}$ Another factor is ixekizumab was only approved on December 1, 2017, ${ }^{7}$ and guselkumab was only approved on July 13 , $2017,{ }^{8}$ which may have skewed the number of ixekizumab and guselkumab prescriptions to a lower frequency because the study collected charts that began on December 5th, 2016. Nonetheless, although ixekizumab and guselkumab were approved last, the number of prescriptions for these two treatments still surpass those of infliximab, brodalumab and etanercept. Data on compliance were not collected, which may influence clinical outcomes or biologic treatment preference. We were also unable to obtain information regarding the tiering system of each insurance company, which would have provided information about how each individual insurer affects choice in biologic. Also, data were not collected on any fees charged by Mount Sinai for injections or subcutaneous injection training. Lastly, some patients may have had a copayment assistance program, in which the drug company would have covered any pharmacy benefit out-of-pocket expenses, thus, newer biologics could be obtained for very little costs. Insurance company coverage would, in turn, not have affected the patient's prescription.

\section{CONCLUSION}

The results of our study highlight certain patterns and discrepancies among biologic prescriptions for Mount Sinai psoriasis patients. Though prescribed biologics vary among insurers, ustekinumab was the most 
frequently prescribed biologic among all insurers, though it is not the most efficacious based on PASI responses. Future research should be conducted to assess how differences in insurance policies (e.g. cost to patient) affect biologic prescribing.

\section{Conflict of Interest Disclosures: None}

Funding: None

\section{Corresponding Author:}

Christopher J. Yao, MPH

University of Rochester School of Medicine and Dentistry, 601 Elmwood Avenue, Box 62, Rochester, NY, 14642

Phone: 917-817-2241

Email: chrisyao@sas.upenn.edu

\section{References:}

1. World Health Organization. Global report on Psoriasis. (2016). World Health Organization website.

http://apps.who.int/iris/bitstream/handle/10665/20 4417/9789241565189 eng.pdf?sequence $=1$.

2. Rachakonda, T. D., Schupp, C. W., \& Armstrong, A. W. (2014). Psoriasis prevalence among adults in the United States. J Am Acad Dermatol, 70(3), 512-516.

3. Kaushik, S. B., \& Lebwohl, M. G. (2019). Psoriasis: Which therapy for which patient psoriasis comorbidities and preferred systemic agents. J Am Acad Dermatol, 80(1), 27-40

4. Heisler, M., Langa, K. M., Eby, E. L., Fendrick, A. M., Kabeto, M. U., \& Piette, J. D. (2004). The Health Effects of Restricting Prescription Medication Use Because of Cost [PDF]. Med Care, 42(7), 626-634.

5. Yao, C. J., \& Lebwohl, M. G. (2019). Onset of Action of Antipsoriatic Drugs for Moderate-toSevere Plaque Psoriasis: An Update. J Drugs Dermatol: JDD, 18(3), 229-233.

6. Food and Drug Administration. Siliq Risk Evaluation and Mitigation Strategy (REMS). https://www.accessdata.fda.gov/drugsatfda docs /rems/Siliq 2017-06-08 Full.pdf. (Publication No. 4109073). (2017, June).

7. Doolen, J. National Psoriasis Foundation. FDA approves Taltz for psoriatic arthritis. National Psoriasis Foundation website. https://www.psoriasis.org/advance/fda-approvestaltz-psoriatic-arthritis. (2017, December 1).

8. National Psoriasis Foundation. FDA approves biologic Tremfya for psoriasis. National Psoriasis Foundation website. https://www.psoriasis.org/advance/fda-approvesbiologic-tremfya-psoriasis. (2017, July 13).

9. Kaushik, S. B., \& Lebwohl, M. G. (2019). CME Part II Psoriasis: Which Therapy for Which Patient Focus on special populations and chronic infections. J Am Acad Dermatol, 80(1), 43-53.

10. Nelson, A. A., Pearce, D. J., Fleischer, A. B., Jr., Balkrishnan, R., \& Feldman, S. R. (2008). Costeffectiveness of biologic treatments for psoriasis based on subjective and objective efficacy measures assessed over a 12-week treatment period. J Am Acad Dermatol, 58(1), 125-135.

11. Sawyer, L. M., Cornic, L., Levin, L. A., Gibbons, C., Moller, A. H., \& Jemec, G. $\quad$ B. (2019). Long-term efficacy of novel therapies in moderate-to-severe plaque psoriasis: a systematic review and network meta-analysis of PASI response. J Eur Acad Dermatol Venereol, 33, 355-366.

12. Lasch K, Liu S, Ursos L, et al. Gastroenterologists' perceptions regarding ulcerative colitis and its management: results from a large-scale survey. Adv Ther. 2016;33:1715-1727. 